\title{
Long term behaviour of the double-mode high-amplitude $\delta$ Scuti star RV Arietis ${ }^{\star}$
}

\author{
M. D. Pócs, B. Szeidl, and G. Virághalmy
}

Konkoly Observatory of the Hungarian Academy of Sciences, 1525 Budapest, PO Box 67, Hungary

Received 30 April 2002 / Accepted 10 July 2002

\begin{abstract}
Stability of both the fundamental and first overtone oscillations of RV Ari was investigated by different methods which led to concordant results. The fundamental period of RV Ari has been decreasing very slowly with a rate of $\left(1 / P_{0}\right)\left(\mathrm{d} P_{0} / \mathrm{d} t\right)=-0.6 \times 10^{-8} \mathrm{y}^{-1}$ while the first overtone period has been increasing a little faster with a rate of $\left(1 / P_{1}\right)\left(\mathrm{d} P_{1} / \mathrm{d} t\right)=+0.9 \times 10^{-8} \mathrm{y}^{-1}$, if we assume linear period changes over the past 65 years. Although the very slow change in the periods is consistent with the evolutionary theories, the fact that the signs of the period changes for the two modes are opposite indicates that the dominating part of the period variation may be the result of non-evolutionary effects. The amplitudes of the fundamental and overtone oscillations have undergone small changes (several mmag) on a time scale of years, and a slight increase in the amplitude of the first overtone pulsation is suspected. An additional frequency at $f_{2}=13.6116 \mathrm{~cd}^{-1}$ is found.
\end{abstract}

Key words. stars: oscillations - stars: variables: $\delta$ Sct - stars: individual: RV Ari

\section{Introduction}

The latest evolutionary models of $\delta$ Scuti stars in the main sequence (MS) or post main sequence (PMS) evolutionary stage (Breger \& Pamyatnykh 1998) predict very slow if any evolutionary period changes. Although in most cases the observed slow changes are not in contradiction with the theoretical prediction, non-evolutionary effects may also generate period variation. The number of high-amplitude $\delta$ Scuti stars (HADS) investigated up to now is, however, fairly small and therefore the study of period changes of a star of this type not yet investigated from this point of view is interesting.

The study of period changes of double-mode highamplitude $\delta$ Scuti stars raises a further important question: how does the overtone frequency change? From a simple theoretical consideration we would guess that the fundamental and overtone frequencies of radially pulsating HADS change at about the same rate and in the same direction. In the two wellinvestigated stars, AI Vel (Walraven et al. 1992) and AE UMa (Pócs \& Szeidl 2001; Zhou 2001), however, the fundamental and overtone frequencies change neither at the same rate nor in the same direction.

The amplitude variation of $\delta$ Scuti stars is also a much discussed question. Recently Breger (2000a) found strong variability in the amplitudes of the dominant modes of the

\footnotetext{
Send offprint requests to: B. Szeidl, e-mail: szeidl@konkoly.hu

* Table 2 is only available in electronic form at http://www . edpsciences.org
}

low-amplitude $\delta$ Scuti star $4 \mathrm{CVn}$. The variability may increase by $\sim 40$ per cent over a decade. It was also shown that the light curve of the medium amplitude $\delta$ Scuti stars AN Lyn (Rodríguez et al. 1997) and V1162 Ori (Arentoft et al. 2001a, 2001b) underwent amplitude changes. The HADS may also be characterized by amplitude changes (e.g. AI Vel: Walraven et al. 1992).

The double-mode high-amplitude $\delta$ Scuti star RV Arietis has not yet been investigated from the point of view of frequency and amplitude changes and this fact gives a reason for a thorough scrutiny of the star.

The variability of RV Ari $\left(\alpha_{2000}=02^{\mathrm{h}} 15^{\mathrm{m}} 08^{\mathrm{s}}, \delta_{2000}=\right.$ $+18^{\circ} 04^{\prime} .4$; 267.1934; GSC 1217 1057) was discovered by Hoffmeister (1934) on Sonneberg plates. Broglia \& Pestarino (1955) derived the dominant period of the variable: $P_{0}=$ 0.09312819 days and came to the conclusion that the light curve of the star was strongly modulated by a second period. Detre (1956) used Broglia \& Pestarino's observations and determined the modulation period: $P_{\mathrm{m}}=0.316256$ days.

RV Ari is a normal Population I HADS with near-solar abundance and it is very close to the end of the main sequence evolutionary stage (Rodríguez et al. 1992; Rodríguez et al. 2000).

In the following sections we give an account of the photometry carried out at the Konkoly Observatory and a summary of all published data. Then we present a detailed analysis of the available data and draw some conclusions about the stability of pulsation of the star. 


\section{The data}

The first observations of the star were made by Lange (1935) and Guriev (1938) visually. They reported 8 and 10 times of light maxima, respectively. Guriev's 355 visual observations obtained on 59 nights in 1936 were published by Soloviev \& Shakhovskoj (1958) two decades later.

During two seasons in 1935 and 1936 Detre (1937) carried out photographic photometry of RV Ari. Balázs (1956) observed the star photographically again in the years 1951-1956 and rediscussed Detre's observations. She published 504 photographic observations obtained on 25 nights and derived 28 times of light maxima from these observations.

In 1951 at the Abastumani Observatory Alania (1954) commenced the photographic investigation of the colour of RR Lyrae stars at maximum light. In the course of this study he observed RV Ari as well and gave one time of light maximum.

The photoelectric photometry of RV Ari was started by Broglia \& Pestarino (1955) at the Merate Observatory in 1954, and then Broglia $(1958,1961)$ continued the program. During the time frames 1954/55, 1955/56 and 1957/58 altogether 1068 yellow and 697 blue observations were obtained on 25 nights and based on these observations 37 times of light maxima were reported.

Fitch et al. (1966) in their comprehensive study of RR Lyrae stars presented $81 U B V$ observations of RV Ari made on three nights in 1964. From these observations they derived one time of light maximum.

Rodríguez et al. (1992) carried out Strömgren photometry of RV Ari on four nights in 1986, and obtained 140 observations in each colour band. From these very accurate observations 6 times of light maxima could be deduced.

The BAV group has kept RV Ari on the observing programme (Agerer et al. 1996, 1998a,b, 1999, 2000, 2001) and published a number of times of light maxima observed photoelectrically or by CCD camera during the last decade. Previously the star was observed visually and photographically by members of the group (Hübscher et al. 1989, 1992); these observations are, however, not accurate enough and are left out of consideration in our discussion.

The photometry made at the Konkoly Observatory well supplements the published observations. The photoelectric observations were started with the Observatory's $60 \mathrm{~cm}$ Newton telescope in 1958 and later the $50 \mathrm{~cm}$ Cassegrain telescope was also used at the Observatory's mountain station. The star was faint for these telescopes and only $B V$ photometry was carried out. $B V(R I)_{C}$ observations were obtained with the Observatory's $1 \mathrm{~m}$ RCC telescope in 1996 and 1997. Throughout our photometry GSC 12170952 was used as a comparison star. Broglia \& Pestarino (1955) used this star (labelled " $b$ " in their paper) as a check star to their comparison star (labelled "a") GSC 12170628 and gave the $V$ magnitudes and $B-V$ colours for these stars as $10.88,+0.66$ and 12.30 , +0.54 respectively. During two observing seasons they compared the two stars several times and found that the magnitude between them were constant within the accuracy of observations: $\Delta V=1.421 \pm 0.005, \Delta B=1.305 \pm 0.004$ in $1954 / 55$
Table 1. Summary of the Konkoly photoelectric photometry.

\begin{tabular}{ccccccc}
\hline \hline Year & Telescope & $\begin{array}{c}\text { Number } \\
\text { of } \\
\text { nights }\end{array}$ & \multicolumn{4}{c}{$\begin{array}{c}\text { Number } \\
\text { of } \\
\text { observations }\end{array}$} \\
& & & $B$ & $V$ & $R$ & $I$ \\
\hline 1958 & $60 \mathrm{~cm}$ Newton & 7 & 214 & 217 & - & - \\
1961 & $60 \mathrm{~cm}$ Newton & 2 & 119 & 115 & - & - \\
1969 & $60 \mathrm{~cm}$ Newton & 1 & 73 & 76 & - & - \\
1972 & $50 \mathrm{~cm}$ Cassegrain & 2 & 134 & 135 & - & - \\
1973 & $50 \mathrm{~cm}$ Cassegrain & 1 & 132 & 129 & - & - \\
1975 & $60 \mathrm{~cm}$ Newton & 3 & 68 & 66 & - & - \\
1996 & $100 \mathrm{~cm}$ RCC & 3 & 205 & 210 & 210 & 204 \\
1997 & $100 \mathrm{~cm}$ RCC & 8 & 380 & 377 & 332 & 318 \\
\hline
\end{tabular}
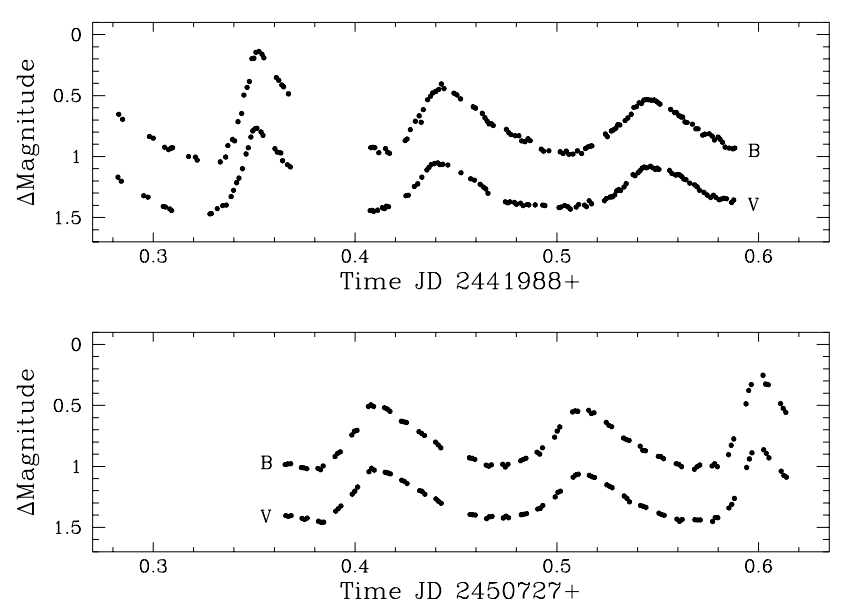

Fig. 1. Two sample light curves observed at Konkoly observatory with the $50 \mathrm{~cm}$ Cassegrain (upper panel) and $100 \mathrm{~cm} \mathrm{RCC} \mathrm{(lower} \mathrm{panel)}$ telescopes.

and $\Delta V=1.424 \pm 0.005$ and $\Delta B=1.312 \pm 0.005$ in $1955 / 56$ (Broglia \& Pestarino 1955; Broglia 1958).

A brief summary of the Konkoly photometry is given in Table 1. Detailed description of the telescopes and photometers used can be found in the paper of Szeidl et al. (2001). The observations will be published in the Communications of the Konkoly Observatory.

Two sample light curves of Konkoly photometry are shown in Fig. 1.

The error of observations can be judged from the light curves (usually from the more or less linear part of the descending branch and minimum). The uncertainity of visual observations is too large and they can only be used with caution; their error exceeds $0.1 \mathrm{mag}$. Both the photographic (Konkoly) and the photoelectric observations (Merate, Sierra Nevada, Konkoly) are suitable for analysis, their errors are less than 0.05 and 0.02 mag, respectively.

From the Konkoly photoelectric photometry 48 new times of light maxima could be derived. These new epochs together with those found in the literature are presented in Table 2.

The accuracy of the times of observed maximum light can be estimated from how the $\mathrm{O}-\mathrm{C}$ values trace out the modulation cycle of 0.005 day amplitude. Since the visual O-C's are scattered randomly around the mean value and do not follow the modulation cycle, their error should be larger than 0.01 day. 
The photographic and photoelectric $\mathrm{O}-\mathrm{C}$ values clearly trace out the modulation cycle and we estimate the errors of times of photographically and photoelectrically observed maximum light to be less than 0.002 and 0.001 day.

\section{The analysis}

\subsection{The Fourier analysis}

The most commonly used technique for the investigation of the stability of pulsation of a star is Fourier analysis.

The observations of RV Ari are well distributed over the past six decades. From the observations, 9 groups were formed; usually the observations of several years were pooled in a group in order to increase the accuracy of the determination of the frequencies and amplitudes.

The multifrequency analysis was performed with the MUFRAN (MUltiFRequency ANalysis) program package (Kolláth 1990). MUFRAN is a collection of methods for frequency determination, sine fitting of observational data and graphics routines for visualization of the results.

The first step in the analysis was finding the frequencies of the fundamental $\left(f_{0}\right)$ and first overtone $\left(f_{1}\right)$ pulsation for each group of the data. The results are presented in Table 3. For the photoelectric data the frequencies are the mean values obtained from the yellow and blue observations. In calculating the error of the frequency determination we followed the method of Walraven et al. (1992). The errors have been estimated using the criterion that one standard error in the frequency $\sigma(f)$ should give a difference of not more than $\Delta \Phi$ in phase for the extreme time intervals, where $\Delta \Phi=0.02$ and 0.05 were chosen for the photoelectric and the photographic or visual observations, respectively, in the case of the fundamental frequency. These phase differences can easily be detected from the light curves of high ampitude variables. The error of the overtone frequency was estimated from $\sigma\left(f_{0}\right)$ supposing a simple $\sigma(f) \sim 1 / a$ dependence on the amplitude (Montgomery \& O’Donoghue 1999).

Guriev's observations made in 1936 were not accurate enough and could be used only for determining the fundamental period as no definite peak appeared in the spectrum of visual observations pre-whitened with the fundamental frequency and its multiples.

The observations of the different groups (except the visual observations) were fitted with the formula

$m$ (or) $\Delta m=a_{0}+\sum_{i, j} a_{i, j} \sin \left(2 \pi f_{i, j} t+\varphi_{i, j}\right)$

by least-squares solution, where $a_{i, j}$ and $\varphi_{i, j}$ denote the Fourier amplitudes and phases, while $t$ the time. The $f_{i, j}$ frequencies are linear combinations of the fundamental $\left(f_{0}=1 / P_{0}\right)$ and the first overtone $\left(f_{1}=1 / P_{1}\right)$ frequencies:

$f_{i, j}=\left|i f_{0} \pm j f_{1}\right|$

where the integers $i$ and $j$ are subject to the constraints: $0 \leq i \leq$ 4 and $0 \leq j \leq 1$. The residuals with higher order fits $(i>4$ and $j>1$ ) did not decrease significantly even if the most accurate observations (Rodríguez et al.'s Strömgren photometry, 1996/97 Konkoly observations) were used.
Table 3. Frequencies (in $\mathrm{cd}^{-1}$ ) for different groups of observations.

\begin{tabular}{ccrr}
\hline \hline years & det & $f_{0}$ & $f_{1}$ \\
\hline \multirow{2}{*}{1936} & vis & 10.7378 & \multicolumn{1}{c}{} \\
& & 2 & \\
$1935-1936$ & pg & 10.7377 & 13.8992 \\
& & 2 & 6 \\
$1951-1956$ & pg & 10.73787 & 13.89910 \\
& & 2 & 6 \\
$1954-1957$ & pe & 10.73788 & 13.89911 \\
& & 2 & 6 \\
$1958-1964$ & pe & 10.73790 & 13.89913 \\
& & 1 & 3 \\
$1969-1975$ & pe & 10.73790 & 13.89913 \\
& & 1 & 3 \\
1986 & pe & 10.735 & 13.896 \\
& & 5 & 15 \\
$1996-1997$ & pe & 10.73790 & 13.89914 \\
& & 5 & 15 \\
\hline
\end{tabular}

Table 4. $a_{1,0}$ and $a_{0,1}$ amplitudes for different groups of observations.

\begin{tabular}{|c|c|c|c|c|}
\hline years & $\begin{array}{c}a_{1,0} \\
\text { in yellow }\end{array}$ & $\begin{array}{l}a_{0,1} \\
\text { band }\end{array}$ & $\begin{array}{l}a_{1,0} \\
\text { in blue }\end{array}$ & $\begin{array}{l}a_{0,1} \\
\text { and }\end{array}$ \\
\hline $1935-1936^{1}$ & - & - & $\begin{array}{r}0.298 \\
7\end{array}$ & $\begin{array}{r}0.071 \\
7\end{array}$ \\
\hline $1951-1956^{1}$ & - & - & $\begin{array}{r}0.300 \\
6\end{array}$ & $\begin{array}{r}0.069 \\
6\end{array}$ \\
\hline 1954-1957 & $\begin{array}{r}0.211 \\
3\end{array}$ & $\begin{array}{r}0.058 \\
3\end{array}$ & $\begin{array}{r}0.271 \\
4\end{array}$ & $\begin{array}{r}0.074 \\
4\end{array}$ \\
\hline 1958-1964 & $\begin{array}{r}0.208 \\
4\end{array}$ & $\begin{array}{r}0.058 \\
4\end{array}$ & $\begin{array}{r}0.261 \\
5\end{array}$ & $\begin{array}{r}0.074 \\
5\end{array}$ \\
\hline 1969-1975 & $\begin{array}{r}0.208 \\
4\end{array}$ & $\begin{array}{r}0.064 \\
4\end{array}$ & $\begin{array}{r}0.262 \\
5\end{array}$ & $\begin{array}{r}0.082 \\
5\end{array}$ \\
\hline $1986^{2}$ & $\begin{array}{r}0.221 \\
3\end{array}$ & $\begin{array}{r}0.061 \\
3\end{array}$ & $\begin{array}{r}0.266 \\
4\end{array}$ & $\begin{array}{r}0.079 \\
4\end{array}$ \\
\hline 1996-1997 & $\begin{array}{r}0.211 \\
3\end{array}$ & $\begin{array}{r}0.065 \\
3\end{array}$ & $\begin{array}{r}0.264 \\
4\end{array}$ & $\begin{array}{r}0.078 \\
4\end{array}$ \\
\hline
\end{tabular}

Remarks: ${ }^{1}$ Photographic observations, ${ }^{2}$ Strömgren photometry.

As the amplitudes $a_{1,0}$ and $a_{0,1}$ can give us a good measure of the stability of pulsation, they are given in Table 4 for the different groups of observations. For the calculation of amplitude errors we have used a Monte-Carlo method, assuming that they follow a Gaussian distribution and $\sigma(\mathrm{pe})=0.02$ and $\sigma(\mathrm{pg})=0.05$ mag (Kovács 1995).

Both Tables 3 and 4 prove that the pulsation of the star is fairly stable and we can only expect very slow changes (if any) in its observables.

\subsection{The $\mathrm{O}-\mathrm{C}$ diagram}

The principal advantage of the Fourier analysis is that all the available observations are included in the frequency analysis. If we were to follow possible changes in the frequency, we would have to divide the observations into short intervals (see 


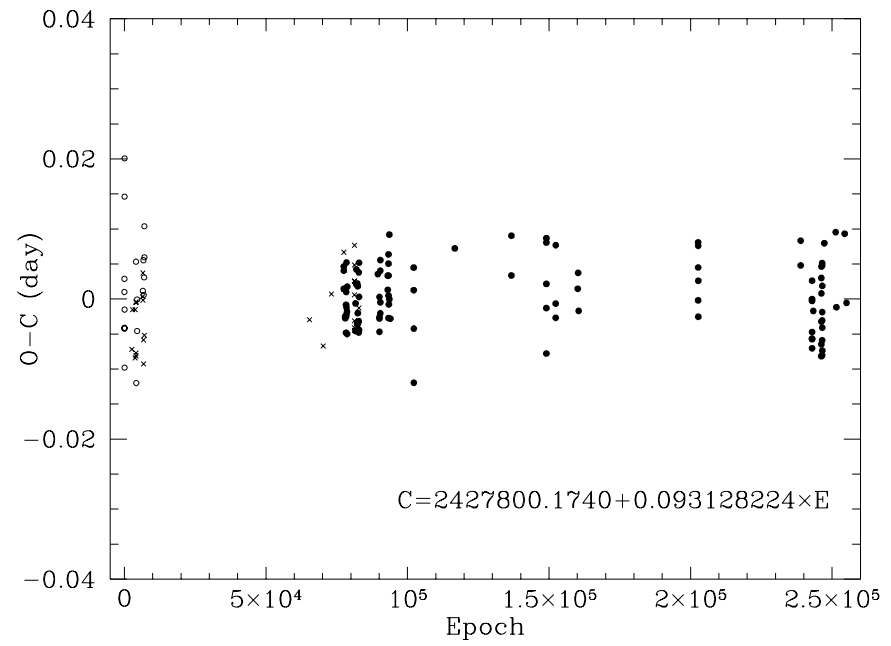

Fig. 2. O-C vs. epoch number diagram for the fundamental mode of RV Ari. Open circles, crosses and dots denote visual, photographic and photoelectric (or CCD) observations, respectively.

Table 3), which results in fairly inaccurate values of the frequency and allows only of a rough estimate of its changes. Moreover, it often happens that the observations are not available, only the times of observed light maxima are reported. The construction of the $\mathrm{O}-\mathrm{C}$ diagram may furnish the dominant frequency and its changes with better accuracy by all accounts.

All the avaible times of light maxima of RV Ari are given in Table 2. The O-C values were calculated with the formula:

$$
C=\mathrm{JD} 2427800.1740+0.093128224 \mathrm{E}
$$

and are presented in Fig. 2. Owing to the double-mode nature of the star, the oscillation of the times of light maxima with the modulation period $P_{\mathrm{m}}$ manifests itself as a scatter on the diagram.

Due to the large uncertainity in the times of light maxima observed visually, only the $\mathrm{O}-\mathrm{C}$ values of the photographic, photoelectric and CCD light maxima were fitted by a quadratic polynomial and the least-squares solution resulted in the following formula ( $n=134$ maxima, time interval is 65 years):

$$
\begin{aligned}
\mathrm{O}-\mathrm{C}= & -(0.0043 \pm .0011)+(6.5 \pm 1.8) \times 10^{-8} E \\
& -(1.4 \pm 0.6) \times 10^{-11} E^{2} .
\end{aligned}
$$

Hence we see that the main (fundamental) period of the star has been decreasing very slowly with the rate of

$$
\frac{1}{P_{0}} \frac{\mathrm{d} P_{0}}{\mathrm{~d} t}=-(1.2 \pm 0.5) \times 10^{-8} \mathrm{y}^{-1}
$$

We should keep in mind that, in case of a double mode HADS, the value of the period change deduced from the $\mathrm{O}-\mathrm{Cs}$ is strongly influenced by the distribution of the observed times of light maxima along the modulation cycle. Therefore the period change rate calculated in this way is rather informative. Moreover the classical O-C method cannot give information about the rate of change in either the modulation or the overtone frequency. Nevertheless, the sharp line at the modulation frequency $f_{\mathrm{m}}=3.16125$ in the Fourier spectrum derived from

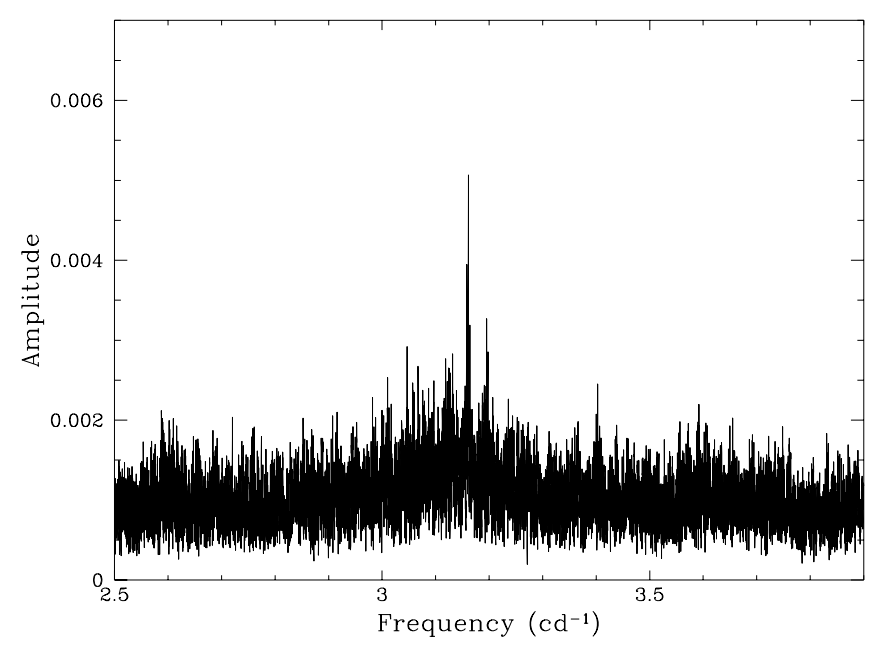

Fig. 3. Fourier spectrum of the photographic, photoelectric and CCD $\mathrm{O}-\mathrm{C}$ values of maximum light.

the $\mathrm{O}-\mathrm{C}$ values (Fig. 3) indicates that the modulation frequency (and consequently the first overtone frequency $f_{1}=f_{0}+f_{\mathrm{m}}$ ) has not changed significantly during the past sixty years.

The Fourier phase method eliminates both shortcomings of the $\mathrm{O}-\mathrm{C}$ method.

\subsection{The Fourier phase diagram}

The Fourier phase method makes the simultaneous investigation of the changes in both the fundamental and overtone frequencies possible. This method was developed for the investigation of frequency changes of double-mode RR Lyrae stars and outlined in the paper of Paparó et al. (1998). The essence of the method is that fixing the amplitudes and frequencies in the Fourier decomposition (in Eqs. (1) and (2)) the variations in the $\varphi_{1,0}$ and $\varphi_{0,1}$ phases reflect the changes in the fundamental and first overtone frequencies. The great advantage of the method is that the complete observed light curves are included in the analysis and so higher accuracy can be achieved, while the reduced number of free parameters makes the use of short segments of observations possible.

Test runs showed that satisfactory results can be obtained if the terms with highest amplitudes are taken into account in Eqs. (1) and (2); in the case of RV Ari the three-frequency $\left(f_{0}, 2 f_{0}, f_{1}\right)$ and the five-frequency $\left(f_{0}, 2 f_{0}, f_{1}, f_{1}-f_{0}, f_{1}+f_{0}\right)$ solutions led to the same results within the limitation of errors. The amplitudes show very small changes, if any, so their mean values were used and the zero point was also treated as a free parameter - as permitted by the Fourier method (Paparó et al. 1998, 2002). Nevertheless the errors of the phase due to the errors in amplitude determination are very small: $\sigma\left(\varphi_{1,0} / 2 \pi\right)=0.3 \sigma_{\mathrm{a}}$ and $\sigma\left(\varphi_{0,1} / 2 \pi\right)=1.8 \sigma_{\mathrm{a}}$ in the case of RV Ari, where $\sigma_{\mathrm{a}}$ is in magnitudes. (This means, for example, that a $0.01 \mathrm{mag}$ error in $a_{1,0}$ results in an error of $0.02 \mathrm{rad}$ in $\left.\varphi_{1,0}.\right)$

The Fourier phase diagram of RV Ari for both frequencies are shown in Fig. 4. The quadratic fits result in the following 


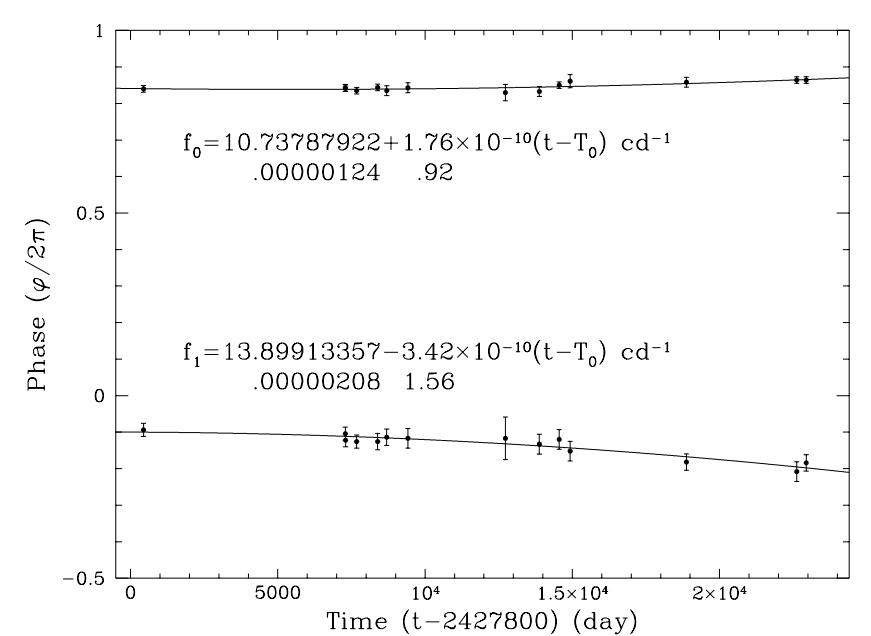

Fig. 4. Fourier phase diagrams for the fundamental frequency $\left(f_{0}\right)$ and for the overtone frequency $\left(f_{1}\right)$. Least-squares quadratic fits are drawn in. The ordinate scales are the phases $\varphi_{1,0} / 2 \pi$ and $\varphi_{0,1} / 2 \pi$ respectively, the abscissa is time in day JD -2427800 .

frequencies:

$$
\begin{aligned}
f_{0}= & (10.7378792 \pm 0.0000012) \\
& +(1.76 \pm 0.92) \times 10^{-10}\left(t-T_{0}\right) \quad \mathrm{cd}^{-1}
\end{aligned}
$$

and

$$
\begin{aligned}
f_{1}= & (13.8991336 \pm 0.0000021) \\
& -(3.42 \pm 1.56) \times 10^{-10}\left(t-T_{0}\right) \quad \mathrm{cd}^{-1}
\end{aligned}
$$

where $T_{0}=\mathrm{JD} 2427800$ and $t$ is given in JD. Hence the period (or frequency) changes are obtained:

$\frac{1}{P_{0}} \frac{\mathrm{d} P_{0}}{\mathrm{~d} t}=-\frac{1}{f_{0}} \frac{\mathrm{d} f_{0}}{\mathrm{~d} t}=(-0.6 \pm 0.3) \times 10^{-8} \mathrm{y}^{-1}$

and

$\frac{1}{P_{1}} \frac{\mathrm{d} P_{1}}{\mathrm{~d} t}=-\frac{1}{f_{1}} \frac{\mathrm{d} f_{1}}{\mathrm{~d} t}=(+0.9 \pm 0.4) \times 10^{-8} \mathrm{y}^{-1}$.

The results obtained by the $\mathrm{O}-\mathrm{C}$ and the Fourier phase methods for the changes in the fundamental period are not in contradiction.

\section{Discussion and conclusions}

\subsection{Amplitudes}

The phenomenon of amplitude variation is quite common among the multimode non-radially pulsating low amplitude $\delta$ Scuti stars. The unevolved, main-sequence $\delta$ Scuti variable XX Pyx can show dramatic amplitude changes on the time scale of months (Handler et al. 1998), while the evolved $\delta$ Scuti star $4 \mathrm{CVn}$ can change its amplitude only on a much longer time scale (several years). Also the variability is very strong: the amplitude may increase by 40 per cent over a decade (Breger 2000a). It is interesting to note that the relative amplitude changes of FG Vir, which is in an evolutionary state between XX Pyx and 4 CVn, are smaller (Breger et al. 1998).
It is also an interesting fact that the well-investigated medium- and high-amplitude $\delta$ Scuti stars have shown detectable amplitude changes. Rodríguez et al. (1997) confirmed that the amplitude of the main frequency of AN Lyn varies on a long time scale of several years, and changes in the amplitude are also present in the secondary frequencies. Arentoft et al. (2001a, 2001b) investigated the interesting medium amplitude $\delta$ Scuti star V1162 Ori and arrived at the remarkable result that the amplitude of the main frequency varies in a very complex way. Irregular variations are superimposed on a cyclic variation. A possible feature is that there are short intervals of constant amplitude in between the large amplitude variations. The other frequencies (except one) have also variable amplitudes. Walraven et al. (1992) found that the amplitudes of the fundamental and first overtone pulsation of AI Vel were significantly larger in the past and they have begun to increase once more. The amplitude changes of the other high-amplitude $\delta$ Scuti star AE UMa were investigated by Pócs \& Szeidl (2001) and Zhou (2001). They found only small changes at the level of several mmag. Zhou also noted that the changes in the amplitudes of the fundamental and first overtone took place in opposite phases, that is, the amplitude of the fundamental mode decreased when that of the first overtone increased.

The available photoelectric observations of RV Ari cover 43 years and are suitable for investigation of long-term variability in the amplitudes. The changes in the amplitude of the fundamental and first overtone were examined by comparing the least-squares amplitude solution for each group of observations, in both $B$ and $V$. The amplitudes $a_{1,0}$ and $a_{0,1}$ can be determined with adequate accuracy and are given in Table 4. There are certainly changes in the amplitudes on a level of several mmag and even a slight increase in the amplitude of the first overtone can be suspected. The photoelectric observations obtained in the years 1954, 1955, 1957, 1958, 1996 and 1997 are fairly numerous, thus the amplitudes can be determined separately for each of these years (with slightly higher errors). The results confirm the above mentioned statement. The amplitude of the fundamental and first overtone oscillations of RV Ari have undergone small changes (several mmag) on a time scale of years, and the amplitude of the overtone oscillation was slightly larger in 1996-1997 than in 1954-1958.

Now it seems certain that the well-studied multiperiodic radial HADS show amplitude variability (Breger 2000b). On the other hand, as Rodríguez (1999) pointed out the monoperiodic HADS do not show any evidence for long-term amplitude variability.

As regards the amplitude of the phase oscillation of light maxima, there is a remarkable and interesting difference between AE UMa and RV Ari. The amplitude of phase modulation of RV Ari (0.00495 days $\approx 7.1 \mathrm{~min})$ is almost twice as large as that of AE UMa (0.00258 days $\approx 3.7 \mathrm{~min})$. Obviously the different behaviour can be explained by the fact that the overtone oscillation is much stronger in RV Ari than in AE UMa. The ratios of the first overtone to the fundamental mode amplitude are: $a_{0,1} / a_{1,0}=0.29$ for RV Ari and $a_{0,1} / a_{1,0}=0.17$ for AE UMa. 


\subsection{Frequencies}

The Fourier analysis of the different groups of observations (Table 3) already suggests that the pulsation modes, both the fundamental and first overtone frequencies of RV Ari have high stability. The Fourier phase method, however, has rendered the determination of more accurate values of period changes possible. If we assume that the periods have changed continuously, the Fourier phase diagrams are fitted by quadratic polynomials. The results show that the fundamental period of RV Ari has decreased very slowly; its relative change has been $(-0.6 \pm 0.3) \times 10^{-8} \mathrm{y}^{-1}$ and even within a $2 \sigma$ level it can be regarded as constant. The first overtone period has changed a little faster, its relative increase has been $(+0.9 \pm 0.4) \times 10^{-8} \mathrm{y}^{-1}$. As a matter of fact these values are not in contradiction with the evolutionary theory, but the changes in opposite directions make the evolutionary interpretation questionable. The situation becomes more interesting if we compare the period changes of RV Ari, AE UMa and AI Vel. AE UMa and AI Vel have constant fundamental periods (within the limitation of error), but their first overtone periods showed significant change. The relative change in the first overtone period of AI Vel is $15 \times 10^{-8} \mathrm{y}^{-1}$ (Walraven et al. 1992) and that of AE UMa is around $-6 \times 10^{-8} \mathrm{y}^{-1}$ (Pócs \& Szeidl 2001; Zhou 2001). In this respect it is worth mentioning that the double-mode RR Lyrae stars show a similar period behaviour. The fundamental and first overtone frequencies of an RRd star may also change in the opposite direction (Paparó et al. 1998). This behaviour may be a general feature of radially oscillating double-mode stars and is difficult to reconcile with evolutionary theories. The change in the period of the fundamental and the overtone mode in opposite directions proves that the dominating part of the period variation should be the result of non-evolutionary effects (Breger 2000b).

We have to admit that the Fourier phase diagrams can equally well be fitted by straight lines. If this is the case, the periods have been subject to small sudden changes between the intervals of constant periods. In this case the above discussion still holds as the periods change in opposite directions.

Broglia (1958) already noted that the residuals of the magnitudes at light maxima were too large and suggested that a third frequency, $f=3.6292 \mathrm{~cd}^{-1}(P=0.27554$ days $)$ was also present in the star's pulsation. The residuals of the least-squares solution for both Rodríguez et al.'s (1992) and the Konkoly data were also somewhat larger than one would expect from the accuracy of the photometries. Further linear combinations of the fundamental and overtone frequencies $(i>4, j>1)$ taken into account could not significantly decrease the residuals. A natural explanation would be that an additional, third frequency, independent of the fundamental and first overtone, was present in the star's oscillation.

The existence of additional frequencies in other HADS has been already demonstrated (Garrido \& Rodríguez 1996; Musazzi et al. 1998; Walraven et al. 1992) and this behaviour might be a common phenomenon among these stars. The importance of detecting the other pulsation modes in this type of star is that the larger the number of identified modes, the greater
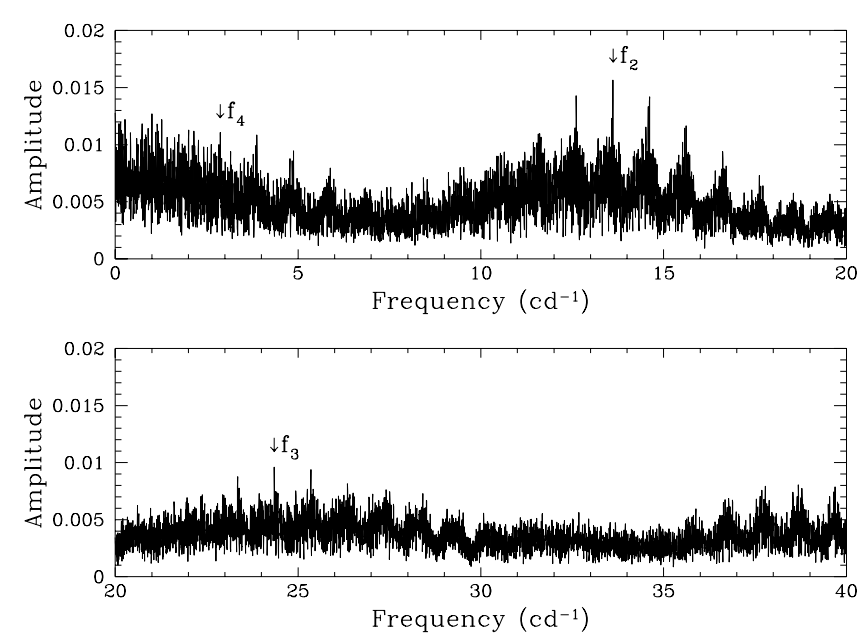

Fig. 5. Fourier spectrum of the Merate $V$ observations between $0-20 \mathrm{~cd}^{-1}$ and $20-40 \mathrm{~cd}^{-1}$ prewhitened with the fundamental frequency $\left(f_{0}\right)$ and its multiplets $\left(2 f_{0}, 3 f_{0}, 4 f_{0}\right)$, with the first overtone frequency $\left(f_{1}\right)$ and its first harmonic $\left(2 f_{1}\right)$ and with the linear combinations of $f_{0}$ and $f_{1}\left(f_{1}-f_{0}, f_{1}+f_{0}, 2 f_{0}+f_{1}, 2 f_{0}-f_{1}, 3 f_{0}-f_{1}, 2 f_{1}-f_{0}\right)$. The peak at $f_{2}=13.61159 \mathrm{~cd}^{-1}$ relates to an additional frequency. $f_{3}=24.34947 \mathrm{~cd}^{-1}$ and $f_{4}=2.87375 \mathrm{~cd}^{-1}$ can be identified with $f_{2}+f_{0}$ and $f_{2}-f_{0}$ respectively.

the number of constraints imposed on pulsation and evolution theory.

Only the fairly numerous and homogeneous Merate observations (Broglia \& Pestarino 1955; Broglia 1958, 1961) can be used in a more rigorous frequency analysis of RV Ari. Figure 5 shows the Fourier spectrum of the Merate $V$ observations prewhitened with the fundamental and first overtone frequencies and their linear combinations. The discussed observations were made over four years. Besides the daily and yearly aliases, the monthly alias also appears as the observations were carried out mainly on moonless nights. The instrumental drifts, small night-to-night changes in the transformation coefficients, changing colour extinctions etc. appear as zero point shifts and result in increased noise-level at the short frequencies. From other parts of the spectrum (around $f=8,19-20,30-35 \mathrm{~cd}^{-1}$ ) we judge the noise-level to be about 5 mmag.

There is no conspicious peak at $f=3.63 \mathrm{~cd}^{-1}$, so Broglia's suggestion should be rejected. (Note that the Fourier spectrum of the $\mathrm{O}-\mathrm{C}$ values of maximum light does not prove the reality of this frequency either, see Fig. 3.) Nevertheless, the existence of the frequency at $f_{2}=13.61159 \mathrm{~cd}^{-1}$ with an amplitude of $16 \mathrm{mmag}$ seems to be real. The reality of this frequency is supported by the clear appearance of the peaks at $f_{3}=24.34947 \mathrm{~cd}^{-1}$ and $f_{4}=2.87375 \mathrm{~cd}^{-1}$ that can be identified with $f_{2}+f_{0}$ and $f_{2}-f_{0}$, respectively.

$f_{0}$ and $f_{1}$ are the radial fundamental and first overtone frequencies (Balona \& Evers 1999), therefore $f_{2}$, which is close to $f_{1}$, should be a non-radial mode. The peaks at the shorter frequencies may be the aftermath of zero point shifts in the observations, but the existence of real frequencies cannot be precluded either. After pre-whitening with the frequency $f_{2}=$ $13.61159 \mathrm{~cd}^{-1}$ a number of frequencies at 5-10 mmag level appear in the spectrum. 
To get a more reliable picture of the multimode pulsation of RV Ari, high quality data from multisite campaigns are needed. The severe zero point shift and alias problems can be overcome only by such data. By all accounts RV Ari is a very interesting star and deserves further study.

Acknowledgements. The authors are indebted to Mr. K. C. Barlay and Dr. L. Szabados for help during the preparation of this paper. They are also grateful to the referee, Dr. T. Arentoft, for helpful comments and valuable suggestions. This work was supported by the Hungarian Research Grant OTKA T-030954 and T-030955.

\section{References}

Agerer, F., Dahm, M., \& Hübscher, J. 1999, IBVS, No. 4712 Agerer, F., Dahm, M., \& Hübscher, J. 2001, IBVS, No. 5017

Agerer, F., \& Hübscher, J. 1996, IBVS, No. 4382

Agerer, F., \& Hübscher, J. 1998a, IBVS, No. 4562

Agerer, F., \& Hübscher, J. 1998b, IBVS, No. 4606

Agerer, F., \& Hübscher, J. 2000, IBVS, No. 4912

Alania, I. F. 1954, Astr. Circ., 146, 14

Arentoft, T., Sterken, C., Handler, G., et al. 2001a, A\&A, 374, 1056

Arentoft, T., Sterken, C., Knudsen, M. R., et al. 2001b, A\&A, 378, L33

Balázs, J. 1956, Mitt. Sternwarte Budapest, No. 40, 8

Balona, L. A., \& Evers, E. A. 1999, MNRAS, 302, 349

Breger, M. 2000a, MNRAS, 313, 129

Breger, M. 2000b, in ASP Conf. Ser. 210, ed. M. Breger, \& M. H. Montgomery, 3

Breger, M., \& Pamyatnykh, A. A. 1998, A\&A, 332, 958

Breger, M., Zima, W., Handler, G., et al. 1998, A\&A, 331, 271

Broglia, P. 1958, Mem. Soc. Astr. It., 29, 113

Broglia, P. 1961, Mem. Soc. Astr. It., 32, 7

Broglia, P., \& Pestarino, E. 1955, Mem. Soc. Astr. It., 26, 429
Detre, L. 1937, Astron. Nachr., 262, 81

Detre, L. 1956, Mitt. Sternwarte Budapest, No. 40, 3

Fitch, W. S., Wiśniewski, W. Z., \& Johnson, H. L. 1966, Commun. Lunar and Planetary Lab., No. 71

Garrido, R., \& Rodríguez, E. 1996, MNRAS, 281, 696

Guriev, N. I. 1938, Tadjik Circ., No. 38

Handler, G., Pamyatnykh, A. A., Zima, W., et al. 1998, MNRAS, 295, 377

Hoffmeister, C. 1934, Astron. Nachr., 253, 195

Hübscher, J., Agerer, F., \& Wunder, E. 1992, BAV Mitt., No. 60

Hübscher, J., Lichtenknecker, D., \& Wunder, E. 1989, BAV Mitt., No. 52

Kolláth, Z. 1990, Occasional Technical Notes, Konkoly Obs., No. 1, http://www.konkoly.hu/staff/kollath/mufran.html

Kovács, G. 1995, A\&A, 295, 693

Lange, G. A. 1935, Tadjik Circ., No. 4

Montgomery, M. H., \& O’Donoghue, D. 1999, Delta Scuti Star Newslet., 13, 28

Musazzi, F., Poretti, \& E. Covino, S. 1998, PASP, 110, 1156

Paparó, M., Saad, S. M., Szeidl, B., et al. 1998, A\&A, 332, 102

Paparó, M., Szeidl, B., Saad, S. M., Kolláth, Z., \& Abu Elazm, M. S. 2002, A\&A, 391, 633

Pócs, M. D., \& Szeidl, B. 2001, A\&A, 368, 880

Rodríguez, E., Rolland, A., López de Coca, P., García-Lobo, E., \& Sedano, J. L. 1992, A\&AS, 93, 189

Rodríguez, E., González-Bedolla, S. F., Rolland, A., et al. 1997, A\&A, 328,235

Rodríguez, E. 1999, PASP, 111, 709

Rodríguez, E., López-González, M. J., \& López de Coca, P. 2000, ASP Conf. Ser. 210, ed. M. Breger, \& M. H. Montgomery, 499

Soloviev, A. V., \& Shakhovskoj, N. M. 1958, Tadjik Trudy, 7, 45

Szeidl, B., Jurcsik, J., Benkő, J., \& Bakos, G. 2001, Commun. Konkoly Obs., No. 101

Walraven, Th., Walraven, J., \& Balona, L. A. 1992, MNRAS, 254, 59

Zhou, A.-Y. 2001, A\&A, 374, 235 